\section{$\S 38$. Separation Capability of Charged Particles by Helmholtz-Type Direct Energy Converter with Electrodes ${ }^{1)}$}

Ishikawa, M., Nemoto, T. (University of Tsukuba) Tomita, Y.

The separation capability of the charged particles is one of the most important capabilities required for Cusptype direct energy converter (Cusp DEC) of D-3He fusion reactors. The preliminary calculations have, however, shown that the separation is not enough with Cusp DEC because of the large self-induced electric field when the thermal input is as large as $10 \mathrm{MW}\left(8.8 \mathrm{MW} / \mathrm{m}^{2}\right)$. And then an alternative DEC using Helmholtz coils (Helmholtz DEC) is designed with electrodes placed closer to the separation area to eliminate ions and electrons in an earlier stage and to reduced the self-induced electric field, as shown in Fig. 1. Behaviors of ions and electrons in the DEC are studied by numerical analyses with a twodimensional approximation.

When both electrodes in Fig. 1 are grounded, most ions reach the ions collector (anode) with the rate of $99.4 \%$, although some ions reach the electrons collector (cathode) or returned to the entrance of the DEC, while all electrons reach the cathode. The potential distribution and the number of charged particles in the DEC have not changed, indicating that the separation is successful and the method setting electrodes closer is effective to reduce the self-induced electric field.

When the electric potential of the anode is given as $50 \mathrm{kV}, 75 \mathrm{kV}$ and $100 \mathrm{kV}$, and that of the cathode is given as $0 \mathrm{~V},-3 \mathrm{kV}$ and $-5 \mathrm{kV}$, about $95 \%$ of ions can reach the anode at $50 \mathrm{kV}$ and $75 \mathrm{kV}$, and about $90 \%$ reaches at $100 \mathrm{kV}$. Figure 2 depicts trajectories of ions and electrons when the potential of anode and cathode is $100 \mathrm{kV}$ and $-3 \mathrm{kV}$, respectively.

It is concluded that the separation is successful using Helmholtz DEC. It is because the electrodes are placed close to the separation area and the charged particles are collected effectively to separate the ions and electrons.

\section{Reference}

1) Nemoto, T., Ishikawa, M., and Momota, H., Fusion

Engineering and Design.63-64 (2002) 501.

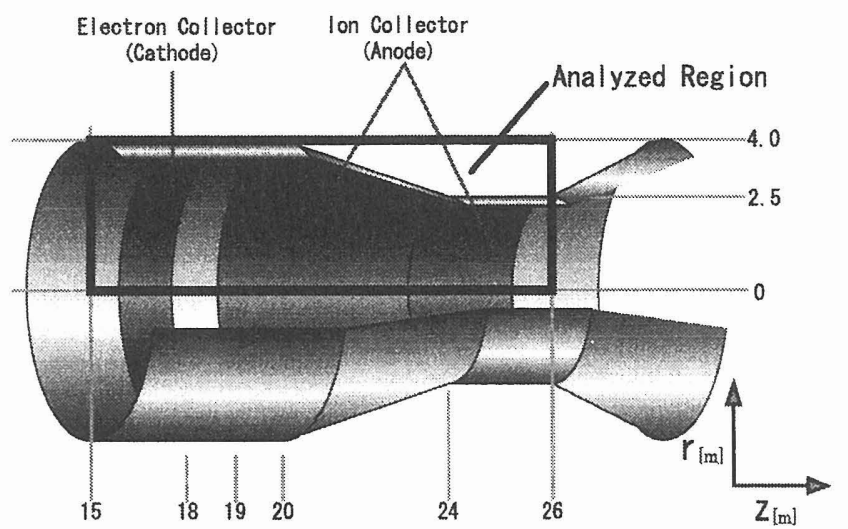

Fig. 1. Helmholtz DEC and analyzed region.

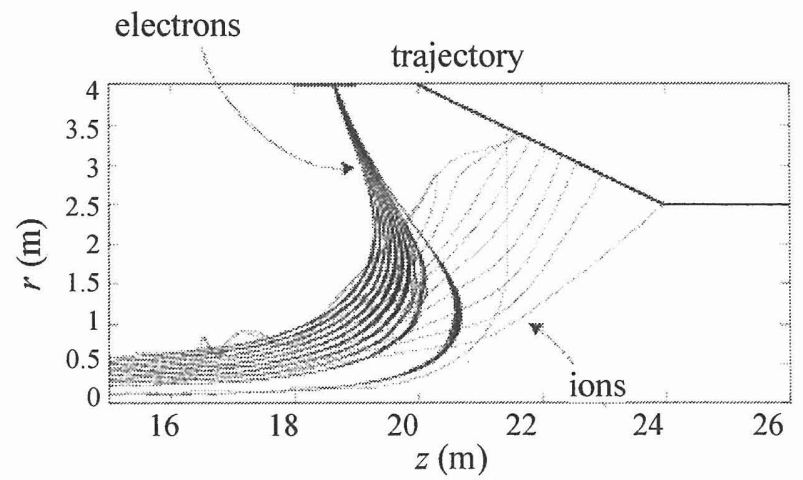

Fig. 2. Trajectory of particles (Helmholtz DEC, anode $100 \mathrm{kV}$, cathode $-3 \mathrm{kV})$. 